\title{
The semistar operations on certain Prüfer domain
}

\author{
Ryûki MATSUDA* \\ in collaboration with Toshitaka Terasaka
}

\begin{abstract}
Let $D$ be a 1-dimensional Prüfer domain with exactly two maximal ideals. We determine the semistar operations on $D$.
\end{abstract}

Let $D$ be an integral domain, let $K$ be its quotient field, let $\mathrm{F}(D)$ be the set of nonzero fractional ideals of $D$, and let $\overline{\mathrm{F}}(D)$ be the set of nonzero $D$-submodules of $K$. A mapping $I \longmapsto I^{\star}$ from $\overline{\mathrm{F}}(D)$ to $\overline{\mathrm{F}}(D)$ is called a semistar operaion on $D$, if it satisfies the following conditions: $(1)(x I)^{\star}=x I^{\star}$ for each $x \in K \backslash\{0\}$ and $I \in \overline{\mathrm{F}}(D)$; (2) $I \subset I^{\star}$ for each $I \in \overline{\mathrm{F}}(D)$; (3) $\left(I^{\star}\right)^{\star}=I^{\star}$ for each $I \in \overline{\mathrm{F}}(D)$; (4) $I \subset J$ implies $I^{\star} \subset J^{\star}$ for each $I, J \in \overline{\mathrm{F}}(D)$. Let $\Sigma^{\prime}(D)$ (resp. $\Sigma(D)$ ) be the set of semistar operations (resp. star operations) on $D$. We have the following,

Theorem $([\mathrm{H}])$. Let $V$ be a valuation domain with maximal ideal $M$.

(1) If $M$ is principal, then $|\Sigma(V)|=1$.

(2) If $M$ is not principal, then $|\Sigma(V)|=2$.

Theorem ([M1]). Let $V$ be an $n$-dimensional valuation domain, let $v$ be a valuation belonging to $V$, and let $\Gamma$ be the value group of $v$. Let $M=P_{n} \supsetneqq P_{n-1} \supsetneqq \cdots \supsetneqq$ $P_{1} \supsetneqq(0)$ be the prime ideals of $V$, and let $H_{n}=\{0\} \varsubsetneqq H_{n-1} \varsubsetneqq \cdots \varsubsetneqq H_{1} \varsubsetneqq \Gamma$ be the convex subgroups of $\Gamma$. Let $m$ be an integer with $n+1 \leq m \leq 2 n+1$. Then the following conditions are equivalent.

(1) $\left|\Sigma^{\prime}(V)\right|=m$.

(2) The maximal ideal of $V_{P_{i}}$ is principal for exactly $2 n+1-m$ of $i$ in $\{1, \cdots, n\}$.

(3) $\Gamma / H_{i}$ has a minimal positive element for exactly $2 n+1-m$ of $i$ in $\{1, \cdots, n\}$.

We want to know $\left|\Sigma^{\prime}(D)\right|$ for Prüfer domains $D$. Let $\Gamma$ be a totally ordered set. If each nonempty subset $S$ of $\Gamma$ which is bounded below has its infimum inf $(S)$ in $\Gamma$, then $\Gamma$ is called complete. In this paper we determine $\left|\Sigma^{\prime}(D)\right|$ for a 1-dimensional Prüfer domain $D$ with two maximal ideals. Our result is the following,

Theorem. Let $D$ be a 1-dimensional Prüfer domain with exactly two maximal ideals $M$ and $N$, and let $\Gamma$ (resp. $\Gamma^{\prime}$ ) be the value group of $D_{M}$ (resp. $\left.D_{N}\right)$.

(1) If both $M$ and $N$ are principal, then $|\Sigma(D)|=1$ and $\left|\Sigma^{\prime}(D)\right|=7$.

Received 13 March, 2010; revised 16 July, 2010.

2000 Mathematics Subject Classification. 13A15.

Key Words and Phrases. semistar operation.

*Professor Emeritus, Ibaraki University (rmazda@adagio.ocn.ne.jp) 
(2) If $M$ is principal, and $\Gamma^{\prime}$ is not complete, then $|\Sigma(D)|=2$ and $\left|\Sigma^{\prime}(D)\right|=14$.

(3) If $\Gamma$ is not complete, and $N$ is principal, then $|\Sigma(D)|=2$ and $\left|\Sigma^{\prime}(D)\right|=14$.

(4) If neither $\Gamma$ nor $\Gamma^{\prime}$ is complete, then $|\Sigma(D)|=9$ and $\left|\Sigma^{\prime}(D)\right|=54$.

In $\S 2$ we will prove (1). In $\S 3$ we will prove (2). In $\S 4$ we will prove (4). The proof for (3) is similar to that of (2). $\S 1$ is the general case.

Throughout the paper, let $D$ be a 1-dimensional Prüfer domain with exactly two maximal ideals $M$ and $N$, let $V=D_{M}$ (resp. $W=D_{N}$ ), let $v$ (resp. $w$ ) be a valuation belonging to $V$ (resp. $W$ ), let $\Gamma$ (resp. $\Gamma^{\prime}$ ) be the value group of $v$ (resp. $w$ ), and let $K$ be the quotient field of $D$. Let $A$ (resp. $B$ ) be the $D$-submodule of $K$ generated by the subset $\left\{\frac{1}{p} \mid p \in M \backslash N\right\}$ (resp. $\left.\left\{\frac{1}{q} \mid q \in N \backslash M\right\}\right)$ of $K$.

This is a continuation of [M2].

\section{$\S 1$ The general case}

Throughout the paper, $p$ denotes an element of $M \backslash N$, and $q$ denotes an element of $N \backslash M$.

(1.1) (1) Each element $x \in D \backslash\{0\}$ can be expressed as 1 or $p$ or $q$ or $p q$ uniquely up to associates, where $p \in M \backslash N$ and $q \in N \backslash M$.

(2) Each element $x \in K \backslash\{0\}$ can be expressed as 1 or $p$ or $q$ or $p q$ or $\frac{1}{p}$ or $\frac{1}{q}$ or $\frac{q}{p}$ or $\frac{p}{q}$ or $\frac{1}{p q}$ uniquely up to associates.

Proof. (1) Let $0 \neq x \in M \cap N$. Choose an element $p \in M \backslash N$. Since $\Gamma$ has rank 1 , there exists a positive integer $n$ such that $v\left(p^{n}\right)>v(x)$. Let $p_{1}=p^{n}+x$. Then we have $v\left(p_{1}\right)=v(x)$ and $p_{1} \in M \backslash N$. Similarly, there exists an element $q_{1} \in N \backslash M$ such that $w\left(q_{1}\right)=w(x)$. Then we have $x=p_{1} q_{1} u$ for a unit $u$ of $D$.

We note that $V=B$ and $W=A$.

A fractional ideal $A_{0} \in \mathrm{F}(D)$ is called of type $\alpha$, if there exists a subset $\left\{p_{\lambda} \mid \Lambda\right\}$ of $M \backslash N$ so that $A_{0}$ is generated by the set $\left\{\frac{1}{p_{\lambda}} \mid \Lambda\right\}, v\left(A_{0}\right)$ is bounded below, and there does not exist inf $v\left(A_{0}\right)$. A fractional ideal $B_{0} \in \mathrm{F}(D)$ is called of type $\beta$, if there exists a subset $\left\{q_{\sigma} \mid \Sigma\right\}$ of $N \backslash M$ so that $B_{0}$ is generated by the set $\left\{\frac{1}{q_{\sigma}} \mid \Sigma\right\}, w\left(B_{0}\right)$ is bounded below, and there does not exist inf $w\left(B_{0}\right)$.

(1.2) Let $I$ be a nonzero fractional ideal of $D$. Then there may arise the following 9-cases:

(1) There exists min $v(I)$, and there exists min $w(I)$;

(2) There exists min $v(I)$, and there exists inf $w(I)$ with inf $w(I) \notin w(I)$;

(3) There exists min $v(I)$, and there does not exist inf $w(I)$;

(4) There exists inf $v(I)$ with $\inf v(I) \notin v(I)$, and there exists min $w(I)$;

(5) There exists inf $v(I)$ with $\inf v(I) \notin v(I)$, and there exists inf $w(I)$ with 
$\inf w(I) \notin w(I)$

(6) There exists $\inf v(I)$ with $\inf v(I) \notin v(I)$, and there does not exist inf $w(I)$;

(7) There does not exist inf $v(I)$, and there exists min $w(I)$;

(8) There does not exist inf $v(I)$, and there exists inf $w(I)$ with $\inf w(I) \notin w(I)$;

(9) There does not exist inf $v(I)$, and there does not exist inf $w(I)$.

Case (1): There exist elements $a, b \in I$ such that $v(a)=\min v(I)$ and $w(b)=$ $\min w(I)$. Let $(a, b)=(c)$ for an element $c \in I$. Then we have $I=(c)$.

Case (2): Assume that $D \varsubsetneqq I$ and $\min v(I)=0$. Let $w\left(\frac{1}{q_{0}}\right)=\inf w(I)$ for an element $q_{0} \in N \backslash M$. Then we have $I=\frac{1}{q_{0}} N$ and $I^{v}=\left(\frac{1}{q_{0}}\right)$.

Let $J$ be a fractional ideal of $D$ such that $I \subset J \subset I^{v}$. Then $J$ is either $I$ or $I^{v}$.

Case (3): Assume that $D \varsubsetneqq I$ and $\min v(I)=0$. Let $\left\{q \in N \backslash M \mid \frac{1}{q} \in I\right\}=$ $\left\{q_{\lambda} \mid \Lambda\right\}$. Then the fractional ideal $\left(\frac{1}{q_{\lambda}} \mid \Lambda\right)$ of $D$ is of type $\beta$, and $I=\left(\frac{1}{q_{\lambda}} \mid \Lambda\right)$. Let $\left\{q_{\sigma} \mid \Sigma\right\}$ be a subset of $N \backslash M$ so that $\left\{w\left(\frac{1}{q_{\sigma}}\right) \mid \Sigma\right\}$ is the lower bounds of $w(I)$. Then we have $I=\cap_{\sigma}\left(\frac{1}{q_{\sigma}}\right)$, hence $I^{v}=I$.

Case (5): Assume that $D \varsubsetneqq I$. Let $v\left(\frac{1}{p_{0}}\right)=\inf v(I)$ for an element $p_{0} \in M \backslash N$, and let $w\left(\frac{1}{q_{0}}\right)=\inf w(I)$ for an element $q_{0} \in N \backslash M$. Then we have $I=\frac{1}{p_{0} q_{0}} M N, I^{v}=$ $\left(\frac{1}{p_{0} q_{0}}\right)$ and $I^{v} \backslash I=\left\{\frac{u}{p_{0} q_{0}}, \frac{q}{p_{0}}, \frac{q}{p_{0} q_{0}}, \frac{p}{q_{0}}, \frac{p}{p_{0} q_{0}} \mid u\right.$ is a unit of $\left.D, p \in M \backslash N, q \in N \backslash M\right\}$.

Let $J$ be a fractional ideal of $D$ such that $I \subset J \subset I^{v}$. Then $J$ is either $I$ or $\left(I, \frac{1}{p_{0}}\right)$ or $\left(I, \frac{1}{q_{0}}\right)$ or $I^{v}$.

Case (6): Assume that $D \varsubsetneqq I$. Let $v\left(\frac{1}{p_{0}}\right)=\inf v(I)$ for an element $p_{0} \in M \backslash N$, and let $\left\{q \in N \backslash M \mid \frac{1}{q} \in I\right\}=\left\{q_{\lambda} \mid \Lambda\right\}$. Then we have $I=\frac{1}{p_{0}} M\left(\frac{1}{q_{\lambda}} \mid \Lambda\right)$, and the fractional ideal $\left(\frac{1}{q_{\lambda}} \mid \Lambda\right)$ of $D$ is of type $\beta$. Let $\left\{q_{\sigma} \mid \Sigma\right\}$ be a subset of $N \backslash M$ so that $\left\{w\left(\frac{1}{q_{\sigma}}\right) \mid \Sigma\right\}$ is the lower bounds of $w(I)$. Then we have $I^{v}=\cap_{\sigma}\left(\frac{1}{p_{0} q_{\sigma}}\right)=\frac{1}{p_{0}}\left(\frac{1}{q_{\lambda}} \mid \Lambda\right)$ and $I^{v} \backslash I=\left\{\frac{u}{p_{0}}, \frac{q}{p_{0}}, \frac{u}{p_{0} q_{\lambda}}, \frac{q}{p_{0} q_{\lambda}} \mid q \in N \backslash M, \lambda \in \Lambda, u\right.$ is a unit of $\left.D\right\}$.

Let $J$ be a fractional ideal of $D$ such that $I \subset J \subset I^{v}$. Then $J$ is either $I$ or $I^{v}$.

Case (9): Assume that $D \varsubsetneqq I$. Let $\left\{p \in M \backslash N \mid \frac{1}{p} \in I\right\}=\left\{p_{\lambda} \mid \Lambda\right\}$, and let $\left\{q \in N \backslash M \mid \frac{1}{q} \in I\right\}=\left\{q_{\sigma} \mid \Sigma\right\}$. Then the fractional ideal $\left(\frac{1}{p_{\lambda}} \mid \Lambda\right)$ of $D$ is of type $\alpha$, the fractional ideal $\left(\frac{1}{q_{\sigma}} \mid \Sigma\right)$ of $D$ is of type $\beta$, and $I=\left(\frac{1}{p_{\lambda}} \mid \Lambda\right)\left(\frac{1}{q_{\sigma}} \mid \Sigma\right)$. Let $\left\{p_{\lambda^{\prime}} \mid \Lambda^{\prime}\right\}$ be a subset of $M \backslash N$ so that $\left\{v\left(\frac{1}{p_{\lambda^{\prime}}}\right) \mid \Lambda^{\prime}\right\}$ is the lower bounds of $v(I)$, and 
let $\left\{q_{\sigma^{\prime}} \mid \Sigma^{\prime}\right\}$ be a subset of $N \backslash M$ so that $\left\{w\left(\frac{1}{q_{\sigma^{\prime}}}\right) \mid \Sigma^{\prime}\right\}$ is the lower bounds of $w(I)$. Then we have $I=\cap_{\lambda^{\prime}, \sigma^{\prime}}\left(\frac{1}{p_{\lambda^{\prime}} q_{\sigma^{\prime}}}\right)$, hence $I^{v}=I$.

Let $A_{0}$ (resp. $B_{0}$ ) be a fractional ideal of $D$ with type $\alpha$ (resp. type $\beta$ ). Then we have $A_{0}=A_{0}^{v}, B_{0}=B_{0}^{v}$ and $A_{0} B_{0}=\left(A_{0} B_{0}\right)^{v}$ by (1.2).

(1.3) Let $A_{0}$ (resp. $B_{0}$ ) be a fractional ideal of $D$ with type $\alpha$ (resp. type $\beta$ ), and let $x \in K \backslash\{0\}$. If either $x M=M$ or $x N=N$ or $x M N=M N$ or $x A_{0} N=A_{0} N$ or $x B_{0} M=B_{0} M$, then $(x)=D$.

(1.4) Example. Let $A_{1}$ (resp. $A_{2}$ ) be the additive group of all integers $\mathbf{Z}$ (resp. all rational numbers $\mathbf{Q}$ ), and introduce on each of them the canonical order. Let $A_{1} \oplus A_{2}$ be their direct sum with the lexicographic order: Let $x=\left(a_{1}, a_{2}\right), y=\left(b_{1}, b_{2}\right)$ be elements of $A_{1} \oplus A_{2}$ with $a_{2}<b_{2}$, then let $x<y$. Let $v_{0}$ (resp. $w_{0}$ ) be the projection mapping of $A_{1} \oplus A_{2}$ to the ordered group $A_{1}$ (resp. $A_{2}$ ). Let $k$ be a field, and let $K$ be the quotient field of the semigroup ring $k\left[X ; A_{1} \oplus A_{2}\right]$. Let $v$ (resp. $w$ ) be the canonical extension of the valuation $v_{0}$ (resp. $w_{0}$ ) on $A_{1} \oplus A_{2}$ to a valuation on $K$, and let $V$ (resp. $W$ ) be the valuation ring on $K$ belonging to $v$ (resp. $w$ ), and let $M^{\prime}$ (resp. $N^{\prime}$ ) be the maximal ideal of $V$ (resp. $W$ ). Let $D=V \cap W$, and let $M=M^{\prime} \cap D$ and $N=N^{\prime} \cap D$. Then we have $V=D_{M}, M^{\prime}=M V, W=D_{N}$ and $N^{\prime}=N W$. We have $\Gamma=\mathbf{Z}$, and $\Gamma^{\prime}=\mathbf{Q}$ is not complete. Let $r$ be a real number with $\mathbf{Q} \not \ngtr r<0$, and le $\left\{q \in N \backslash M \mid w\left(\frac{1}{q}\right)>r\right\}=\left\{q_{\sigma} \mid \Sigma\right\}$. Then the fractional ideal $\left(\frac{1}{q_{\sigma}} \mid \Sigma\right)$ of $D$ has type $\beta$. Let $\left.I=M\left(\frac{1}{q_{\sigma}}\right) \mid \Sigma\right)$. Then there exists inf $v(I)$ with inf $v(I) \notin v(I)$, and there does not exist inf $w(I)$.

(1.5) Let $\star$ be a star operation on $D$. Then we have that $M^{\star}$ is either $M$ or $D, N^{\star}$ is either $N$ or $D$, and $(M N)^{\star}$ is either $M N$ or $M$ or $N$ or $D$. Either $\left(A_{0} N\right)^{\star}=A_{0} N$ for each fractional ideal $A_{0}$ of $D$ with type $\alpha$, or $\left(A_{0} N\right)^{\star}=A_{0}$ for each fractional ideal $A_{0}$ of $D$ with type $\alpha$. Either $\left(B_{0} M\right)^{\star}=B_{0} M$ for each fractional ideal $B_{0}$ of $D$ with type $\beta$, or $\left(B_{0} M\right)^{\star}=B_{0}$ for each fractional ideal $B_{0}$ of $D$ with type $\beta$.

Proof. Suppose that $\left(A_{0}^{1} N\right)^{\star}=A_{0}^{1} N$ and $\left(A_{0}^{2} N\right)^{\star}=A_{0}^{2}$ for some fractional ideals $A_{0}^{1}, A_{0}^{2}$ of $D$ with type $\alpha$. There exists an element $p \in M \backslash N$ such that $A_{0}^{2} \subset \frac{1}{p} A_{0}^{1}$. It follows that $A_{0}^{2} \subset \frac{1}{p} A_{0}^{1} N$; a contradiction.

Let $\star$ be a star operation on $D$. If $\left(A_{0} N\right)^{\star}=A_{0} N$ for each fractional ideal $A_{0}$ of $D$ with type $\alpha$, and $\left(B_{0} M\right)^{\star}=B_{0} M$ for each fractional ideal $B_{0}$ of $D$ with type $\beta$, then we say that $\star$ is of type $(\alpha, \beta)$. If $\left(A_{0} N\right)^{\star}=A_{0} N$ for each fractional ideal $A_{0}$ of $D$ with type $\alpha$, and $\left(B_{0} M\right)^{\star}=B_{0}$ for each fractional ideal $B_{0}$ of $D$ with type $\beta$, then we say that $\star$ is of type $\left(\alpha, \beta^{\prime}\right)$. If $\left(A_{0} N\right)^{\star}=A_{0}$ for each fractional ideal $A_{0}$ of $D$ with type $\alpha$, and $\left(B_{0} M\right)^{\star}=B_{0} M$ for each fractional ideal $B_{0}$ of $D$ with type $\beta$, then we say that $\star$ is of type $\left(\alpha^{\prime}, \beta\right)$. If $\left(A_{0} N\right)^{\star}=A_{0}$ for each fractional ideal $A_{0}$ of $D$ with 
type $\alpha$, and $\left(B_{0} M\right)^{\star}=B_{0}$ for each fractional ideal $B_{0}$ of $D$ with type $\beta$, then we say that $\star$ is of type $\left(\alpha^{\prime}, \beta^{\prime}\right)$.

(1.6) We have $|\Sigma(D)| \leq 64$.

Proof. (1.2) implies that $\left\{I \in \mathrm{F}(D) \mid I \varsubsetneqq I^{v}\right\} \subset\left\{x M, x N, x M N, x A_{0} N, x B_{0} M \mid\right.$ $x \in K \backslash\{0\}, A_{0}$ (resp. $B_{0}$ ) is a fractional ideal of $D$ with type $\alpha$ (resp. type $\beta$ ) $\}$. Let $\star$ be a star operation on $D$. Then we have that $M^{\star}$ is either $M$ or $D, N^{\star}$ is either $N$ or $D$, and $(M N)^{\star}$ is either $M N$ or $M$ or $N$ or $D$. $\star$ has type $(\alpha, \beta)$ or type $\left(\alpha, \beta^{\prime}\right)$ or type $\left(\alpha^{\prime}, \beta\right)$ or type $\left(\alpha^{\prime}, \beta^{\prime}\right)$.

(1.7) Let $I \in \overline{\mathrm{F}}(D) \backslash \mathrm{F}(D)$. There may arise the following 7-cases:

(1) There exists min $v(I)$, and $w(I)=\Gamma^{\prime}$;

(2) $v(I)$ is bounded below, there exists $\inf v(I)$ with $\inf v(I) \notin v(I)$, and $w(I)=$ $\Gamma^{\prime}$

(3) $v(I)$ is bounded below, there does not exist inf $v(I)$, and $w(I)=\Gamma^{\prime}$;

(4) $v(I)=\Gamma$, and there exists min $w(I)$;

(5) $v(I)=\Gamma$, and there exists inf $w(I)$ with inf $w(I) \notin w(I)$;

(6) $v(I)=\Gamma, w(I)$ is bounded below, and there does not exist inf $w(I)$;

(7) $v(I)=\Gamma$, and $w(I)=\Gamma^{\prime}$.

Case (1): Assume that $\min v(I)=0$. Then we have $I=B$.

Case (2): Assume that $v\left(\frac{1}{p_{0}}\right)=\inf v(I)$ for an element $p_{0} \in M \backslash N$. Let $\left\{p \in M \backslash N \mid \frac{1}{p} \in I\right\}=\left\{p_{\lambda} \mid \Lambda\right\}$. Then we have $\left(\frac{1}{p_{\lambda}} \mid \Lambda\right)=\frac{1}{p_{0}} M, I=\frac{1}{p_{0}} M B$, and $M^{v}=D$.

Case (3): Assume that $I \supsetneqq D$. Let $\left\{p \in M \backslash N \mid \frac{1}{p} \in I\right\}=\left\{p_{\lambda} \mid \Lambda\right\}$, and let $A_{0}=\left(\frac{1}{p_{\lambda}} \mid \Lambda\right)$. Then $A_{0}$ is a fractional ideal of $D$ with type $\alpha$, and $I=A_{0} B$.

Case (7): $I=K$.

Let $I^{d^{\prime}}=I$ (resp. $I^{v^{\prime}}=\left(I^{-1}\right)^{-1}, I^{e}=K$ ) for each $I \in \overline{\mathrm{F}}(D)$. Then $\mathrm{d}^{\prime}$ (resp. $\mathrm{v}^{\prime}, \mathrm{e}$ ) is a semistar operation on $D$, and is called the $\mathrm{d}^{\prime}$-operation (resp. $\mathrm{v}^{\prime}$-operation, e-operation) on $D$.

Let $I \subset J$ be an inclusion relation of elements of $\overline{\mathrm{F}}(D)$. A mapping $\star$ from $\overline{\mathrm{F}}(D)$ to $\overline{\mathrm{F}}(D)$ is called monotone for $I \subset J$, if $I^{\star} \subset J^{\star}$.

(1.8) Let $\star$ be a semistar operation on $D$.

(1) $A^{\star}$ is either $A$ or $K . B^{\star}$ is either $B$ or $K$.

(2) $(A N)^{\star}$ is either $A N$ or $A$ or $K .(B M)^{\star}$ is either $B M$ or $B$ or $K$.

(3) If $A^{\star}=A$, then $\left(A B_{0}\right)^{\star}=A B_{0}$ for each fractional ideal $B_{0}$ of $D$ with type $\beta$. If $B^{\star}=B$, then $\left(A_{0} B\right)^{\star}=A_{0} B$ for each fractional ideal $A_{0}$ of $D$ with type $\alpha$.

Proof. (1) Assume that $A \varsubsetneqq A^{\star}$. There exists an element $q \in N \backslash M$ such that $\frac{1}{q} \in A^{\star}$. Then we have $\frac{1}{q} A \subset A^{\star}$, and $A^{\star}=\frac{1}{q} A^{\star}$. Hence $A^{\star}=K$. 
(2) We may assume that $A^{\star}=A$. We have $(A N)^{\star} \subset A$. Let $A N \varsubsetneqq J \subset A$ be a $D$-submodule of $K$. There exists an element $x \in J$ with $w(x)=0$. It follows that $\frac{1}{p_{0}} \in J$ for some $p_{0} \in M \backslash N$. Then we have $D \subset J$ and $\frac{1}{p} \in J$ for each $p \in M \backslash N$. Hence $J=A$.

(3) Suppose that $A B_{0} \varsubsetneqq\left(A B_{0}\right)^{\star}$. There exists an element $q_{1} \in N \backslash M$ such that $A B_{0} \not \ngtr \frac{1}{q_{1}} \in\left(A B_{0}\right)^{\star}$. Then $w\left(\frac{1}{q_{1}}\right)$ is a lower bound of $w\left(B_{0}\right)$, and $A B_{0} \subset A \frac{1}{q_{1}} \subset$ $\left(A B_{0}\right)^{\star}$. There exists an element $q_{2} \in N \backslash M$ with $w\left(\frac{1}{q_{1}}\right)<w\left(\frac{1}{q_{2}}\right)$ so that $w\left(\frac{1}{q_{2}}\right)$ is a lower bound of $w\left(B_{0}\right)$. Then we have $A B_{0} \subset A \frac{1}{q_{2}} \varsubsetneqq A \frac{1}{q_{1}} \subset\left(A B_{0}\right)^{\star}$. Hence $\left(A B_{0}\right)^{\star} \subset A \frac{1}{q_{2}} \varsubsetneqq A \frac{1}{q_{1}} \subset\left(A B_{0}\right)^{\star} ;$ a contradiction.

(1.9) We have $\left|\Sigma^{\prime}(D)\right|<\infty$.

Proof. Let $\Sigma_{0}^{\prime}$ be the set of extensions of star operations on $D$ to semistar operations on $D$. It is sufficient to prove that $\left|\Sigma_{0}^{\prime}\right|<\infty$.

Let $\star$ be an element of $\Sigma_{0}^{\prime}$. Then the restriction $\left.\star\right|_{F(D)}$ belongs to the finite set $\Sigma(D) . A^{\star}$ is either $A$ or $K, B^{\star}$ is either $B$ or $K,(A N)^{\star}$ is either $A N$ or $A$ or $K$, and $(B M)^{\star}$ is either $B M$ or $B$ or $K$.

Let $\star^{\prime}$ be an element of $\Sigma_{0}^{\prime}$ satisfying the following conditions: $\left.\star\right|_{F(D)}=\left.\star^{\prime}\right|_{F(D)}, A^{\star}=$ $A^{\star^{\prime}}, B^{\star}=B^{\star^{\prime}},(A N)^{\star}=(A N)^{\star^{\prime}}$ and $(B M)^{\star}=(B M)^{\star^{\prime}}$. To prove that $\left|\Sigma_{0}^{\prime}\right|<\infty$, it is sufficient to prove that $\star=\star^{\prime}$.

Let $I \in \overline{\mathrm{F}}(D)$. We must show that $I^{\star}=I^{\star^{\prime}}$.

If $I \in \mathrm{F}(D)$, we have $I^{\star}=I^{\star^{\prime}}$, since $\left.\star\right|_{F(D)}=\left.\star^{\prime}\right|_{F(D)}$.

Assume that there exists $\min v(I)$ and that $w(I)=\Gamma^{\prime}$. Then $I=x B$ for some element $x \in K$. Then we have $I^{\star}=x B^{\star}=x B^{\star^{\prime}}=I^{\star^{\prime}}$.

Assume that there exists $\inf v(I)$ with $\inf v(I) \notin v(I)$ and that $w(I)=\Gamma^{\prime}$. Then we have $I=x B M$ for some element $x \in K$. Hence $I^{\star}=I^{\star^{\prime}}$.

Assume that $v(I)$ is bounded, there does not exist inf $v(I)$, and $w(I)=\Gamma^{\prime}$. There exists an element $x \in K$ and a fractional ideal $A_{0}$ with type $\alpha$ such that $x I=A_{0} B$. If $B^{\star}=K$, then $I^{\star}=I^{\star^{\prime}}=K$. If $B^{\star}=B$, then $I^{\star}=I^{\star^{\prime}}=\frac{1}{x} A_{0} B$.

Assume that $v(I)=\Gamma$ and $w(I)=\Gamma^{\prime}$. Then $I=K$. Hence $I^{\star}=I^{\star^{\prime}}$.

The proof is complete.

\section{$\S 2$ The case where both $M$ and $N$ are principal}

This case is just the case of [M2, Proposition 6 (2)]. We will review it for our convenience. Thus, each fractional ideal of $D$ is principal. Hence we have $\Sigma(D)=\{\mathrm{d}\}$.

We have $\overline{\mathrm{F}}(D) \backslash \mathrm{F}(D)=\{x A, x B, K \mid x \in K \backslash\{0\}\}$.

Let $\star$ be a semistar operation on $D$. Then $A^{\star}$ is either $A$ or $K$, and $B^{\star}$ is either $B$ or $K$.

(2.1) (1) Set $I_{0}^{\star}=I_{0}$ for each $I_{0} \in \mathrm{F}(D)$, and set $A^{\star}=A$ and $B^{\star}=B$. Then 
there is determined a semistar operation $\star$ on $D$ uniquely, and $\star=\mathrm{d}^{\prime}$.

(2) Set $I_{0}^{\star}=I_{0}$ for each $I_{0} \in \mathrm{F}(D)$, and set $A^{\star}=A$ and $B^{\star}=K$. Then there is determined a semistar operation $\star$ on $D$ uniquely.

(3) Set $I_{0}^{\star}=I_{0}$ for each $I_{0} \in \mathrm{F}(D)$, and set $A^{\star}=K$ and $B^{\star}=B$. Then there is determined a semistar operation $\star$ on $D$ uniquely.

(4) Set $I_{0}^{\star}=I_{0}$ for each $I_{0} \in \mathrm{F}(D)$, and set $A^{\star}=K$ and $B^{\star}=K$. Then there is determined a semistar operation $\star$ on $D$ uniquely, and $\star=\mathrm{v}^{\prime}$.

(2.2) We have $|\Sigma(D)|=1$ and $\left|\Sigma^{\prime}(D)\right|=7$.

\section{$\S 3$ The case where $M$ is principal and $\Gamma^{\prime}$ is not complete}

Then $N$ is not principal.

(3.1) We have $\left\{I \in \mathrm{F}(D) \mid I \varsubsetneqq I^{v}\right\}=\{x N \mid x \in K \backslash\{0\}\}$.

If $x N \subset N$ for an element $x \in K \backslash\{0\}$, then $x \in D$.

(3.2) (1) Set $N^{\star_{1}}=N$. Then there is determined a star operation $\star_{1}$ on $D$ uniquely, and then $\star_{1}=\mathrm{d}$.

(2) Set $N^{\star_{2}}=D$. Then there is determined a star operation $\star_{2}$ on $D$ uniquely, and then $\star_{2}=\mathrm{v}$.

(3.3) We have $|\Sigma(D)|=2$ and $\Sigma(D)=\{\mathrm{d}, \mathrm{v}\}$.

(3.4) We have $\overline{\mathrm{F}}(D) \backslash \mathrm{F}(D)=\left\{x A, x B, x A N, x A B_{0}, K \mid x \in K \backslash\{0\}, B_{0}\right.$ is a fractional ideal of $D$ with type $\beta$.

(3.5) Let $\star$ be a semistar operation on $D$. Then we have that $A^{\star}$ is either $A$ or $K, B^{\star}$ is either $B$ or $K$, and $(A N)^{\star}$ is either $A N$ or $A$ or $K$. If $A^{\star}=A$, then $\left(A B_{0}\right)^{\star}=A B_{0}$ for each fractional ideal $B_{0}$ of $D$ with type $\beta$.

(3.6) Let $\star_{i}$ be a star operation on $D$.

(1) Set $I_{0}^{\star_{i}^{1}}=I_{0}^{\star_{i}}$ for each $I_{0} \in \mathrm{F}(D)$, and set $A^{\star_{i}^{1}}=A, B^{\star_{i}^{1}}=B$ and $(A N)^{\star_{i}^{1}}=$ $A N$. Then there is determined a unique mapping $\star_{i}^{1}$ from $\overline{\mathrm{F}}(D)$ to $\overline{\mathrm{F}}(D)$.

(2) Set $I_{0}^{\star_{i}^{2}}=I_{0}^{\star_{i}}$ for each $I_{0} \in \mathrm{F}(D)$, and set $A^{\star_{i}^{2}}=A, B^{\star_{i}^{2}}=B$ and $(A N)^{\star_{i}^{2}}=A$. Then there is determined a unique mapping $\star_{i}^{2}$ from $\overline{\mathrm{F}}(D)$ to $\overline{\mathrm{F}}(D)$.

(3) Set $I_{0}^{\star_{i}^{3}}=I_{0}^{\star_{i}}$ for each $I_{0} \in \mathrm{F}(D)$, and set $A^{\star_{i}^{3}}=A, B^{\star_{i}^{3}}=K$ and $(A N)^{\star_{i}^{3}}=$ $A N$. Then there is determined a unique mapping $\star_{i}^{3}$ from $\overline{\mathrm{F}}(D)$ to $\overline{\mathrm{F}}(D)$.

(4) Set $I_{0}^{\star_{i}^{4}}=I_{0}^{\star_{i}}$ for each $I_{0} \in \mathrm{F}(D)$, and set $A^{\star_{i}^{4}}=A, B^{\star_{i}^{4}}=K$ and $(A N)^{\star_{i}^{4}}=A$. Then there is determined a unique mappping $\star_{i}^{4}$ from $\overline{\mathrm{F}}(D)$ to $\overline{\mathrm{F}}(D)$.

(5) Set $I_{0}^{\star_{i}^{5}}=I_{0}^{\star_{i}}$ for each $I_{0} \in \mathrm{F}(D)$, and set $A^{\star_{i}^{5}}=K$ and $B^{\star_{i}^{5}}=B$. Then there is determined a unique mapping $\star_{i}^{5}$ from $\overline{\mathrm{F}}(D)$ to $\overline{\mathrm{F}}(D)$.

(6) Set $I_{0}^{\star_{i}^{6}}=I_{0}^{\star_{i}}$ for each $I_{0} \in \mathrm{F}(D)$, and set $A^{\star_{i}^{6}}=K$ and $B^{\star_{i}^{6}}=K$. Then there 
is determined a unique mapping $\star_{i}^{6}$ from $\overline{\mathrm{F}}(D)$ to $\overline{\mathrm{F}}(D)$.

(3.7) Each $\star_{i}^{j}$ satisfies the following conditions: $(x I)^{\star}=x I^{\star}$ for each $x \in K \backslash\{0\}$ and $I \in \overline{\mathrm{F}}(D) ; I \subset I^{\star}$ for each $I \in \overline{\mathrm{F}}(D)$; and $\left(I^{\star}\right)^{\star}=I^{\star}$ for each $I \in \overline{\mathrm{F}}(D)$.

(3.8) Let $B_{0}$ be a fractional ideal of $D$ with type $\beta$, and let $x \in K \backslash\{0\}$.

If $x N \subset A$, then $(x) \subset A$.

If $x N \subset B$, then $(x) \subset B$.

If $x N \subset A B_{0}$, then $(x) \subset A B_{0}$.

If $x A N \subset A$, then $x A \subset A$.

If $x A N \subset A B_{0}$, then $x A \subset A B_{0}$.

Proof. $x$ is either $u$ or $p$ or $q$ or $p q$ or $\frac{1}{p}$ or $\frac{1}{q}$ or $\frac{q}{p}$ or $\frac{p}{q}$ or $\frac{1}{p q}$, where $p \in$ $M \backslash N, q \in N \backslash M$, and $u$ is a unit of $D$.

(3.9) $\star_{1}^{1}$ is a semistar operation on $D$, and $\star_{1}^{1}=\mathrm{d}^{\prime}$.

$\star_{1}^{2}$ is a semistar operation on $D$.

$\star_{2}^{2}$ is a semistar operation on $D$.

$\star_{1}^{3}$ is a semistar operation on $D$.

$\star_{1}^{4}$ is a semistar operation on $D$.

$\star_{2}^{4}$ is a semistar operation on $D$.

$\star_{1}^{5}$ is a semistar operation on $D$.

$\star_{2}^{5}$ is a semistar operation on $D$.

$\star_{1}^{6}$ is a semistar operation on $D$.

$\star_{2}^{6}$ is a semistar operation on $D$, and $\star_{2}^{6}=\mathrm{v}^{\prime}$.

(3.10) $\star_{2}^{1}$ is not monotone for $N \subset A N . \star_{2}^{3}$ is not monotone for $N \subset A N$.

(3.11) $\left|\Sigma^{\prime}(D)\right|=14$.

$\S 4$ The case where neither $\Gamma$ nor $\Gamma^{\prime}$ is complete

Then neither $M$ nor $N$ is principal.

(4.1) We have $\left\{I \in \mathrm{F}(D) \mid I \varsubsetneqq I^{v}\right\}=\left\{x M, x N, x M N, x A_{0} N, x B_{0} M \mid x \in\right.$ $K \backslash\{0\}, A_{0}$ (resp. $B_{0}$ ) is a fractional ideal of $D$ with type $\alpha$ (resp. type $\beta$ ) $\}$.

(4.2) Let $\star$ be a star operation on $D$. Then we have that $M^{\star}$ is either $M$ or $D$, $N^{\star}$ is either $N$ or $D$, and $(M N)^{\star}$ is either $M N$ or $M$ or $N$ or $D$. $\star$ has the type $(\alpha, \beta)$ or $\left(\alpha, \beta^{\prime}\right)$ or $\left(\alpha^{\prime}, \beta\right)$ or $\left(\alpha^{\prime}, \beta^{\prime}\right)$.

(4.3) Let $A_{0}$ (resp. $B_{0}$ ) be a fractional ideal of $D$ with type $\alpha$ (resp. type $\beta$ ).

(1) Set $M^{\star_{1}}=M, N^{\star_{1}}=N,\left(A_{0} N\right)^{\star_{1}}=A_{0} N$ and $\left(B_{0} M\right)^{\star_{1}}=B_{0} M$. Then there is determined a unique mapping $\star_{1}$ from $\mathrm{F}(D)$ to $\mathrm{F}(D)$.

(2) Set $M^{\star_{2}}=M, N^{\star_{2}}=N,\left(A_{0} N\right)^{\star_{2}}=A_{0} N$ and $\left(B_{0} M\right)^{\star_{2}}=B_{0}$. Then there 
is determined a unique maping $\star_{2}$ from $\mathrm{F}(D)$ to $\mathrm{F}(D)$.

(3) Set $M^{\star_{3}}=M, N^{\star_{3}}=N,\left(A_{0} N\right)^{\star_{3}}=A_{0}$ and $\left(B_{0} M\right)^{\star_{3}}=B_{0} M$. Then there is determined a unique mapping $\star_{3}$ from $\mathrm{F}(D)$ to $\mathrm{F}(D)$.

(4) Set $M^{\star_{4}}=M, N^{\star_{4}}=N,\left(A_{0} N\right)^{\star_{4}}=A_{0}$ and $\left(B_{0} M\right)^{\star_{4}}=B_{0}$. Then there is determined a unique mapping $\star_{4}$ from $\mathrm{F}(D)$ to $\mathrm{F}(D)$.

(5) Set $M^{\star_{5}}=M, N^{\star_{5}}=D$ and $\left(B_{0} M\right)^{\star_{5}}=B_{0} M$. Then there is determined a unique mapping $\star_{5}$ from $\mathrm{F}(D)$ to $\mathrm{F}(D)$.

(6) Set $M^{\star_{6}}=M, N^{\star_{6}}=D$ and $\left(B_{0} M\right)^{\star_{6}}=B_{0}$. Then there is determined a unique mapping $\star_{6}$ from $\mathrm{F}(D)$ to $\mathrm{F}(D)$.

(7) Set $M^{\star_{7}}=D, N^{\star_{7}}=N$ and $\left(A_{0} N\right)^{\star_{7}}=A_{0} N$. Then there is determined a unique mapping $\star_{7}$ from $\mathrm{F}(D)$ to $\mathrm{F}(D)$.

(8) Set $M^{\star_{8}}=D, N^{\star_{8}}=N$ and $\left(A_{0} N\right)^{\star_{8}}=A_{0}$. Then there is determined a unique mapping $\star_{8}$ from $\mathrm{F}(D)$ to $\mathrm{F}(D)$.

(9) Set $M^{\star 9}=D$ and $N^{\star 9}=D$. Then there is determined a unique mapping $\star_{9}$ from $\mathrm{F}(D)$ to $\mathrm{F}(D)$.

(4.4) Let $A_{0}$ (resp. $B_{0}$ ) be a fractional ideal of $D$ with type $\alpha$ (resp. type $\beta$ ), and let $x \in K \backslash\{0\}$.

If $x M \subset D$, then $(x) \subset D$.

If $x M \subset N$, then $(x) \subset N$.

If $x M \subset A_{0} N$, then $(x) \subset A_{0} N$.

If $x M \subset B_{0} M$, then $(x) \subset B_{0}$.

If $x N \subset D$, then $(x) \subset D$.

If $x N \subset M$, then $(x) \subset M$.

If $x N \subset A_{0} N$, then $(x) \subset A_{0}$.

If $x N \subset B_{0} M$, then $(x) \subset B_{0} M$.

If $x M N \subset D$, then $(x) \subset D$.

If $x M N \subset M$, then $x M \subset M$.

If $x M N \subset M$, then $x N \subset D$.

If $x M N \subset N$, then $x M \subset D$.

If $x M N \subset N$, then $x N \subset N$.

If $x M N \subset A_{0} N$, then $(x) \subset A_{0}$.

If $x M N \subset B_{0} M$, then $(x) \subset B_{0}$.

If $x A_{0} N \subset D$, then $x A_{0} \subset D$.

If $x A_{0} N \subset M$, then $x A_{0} \subset M$.

If $x A_{0} N \subset N$, then $x A_{0} \subset N$.

If $x A_{0} N \subset M N$, then $x A_{0} \subset M N$.

If $x A_{0} N \subset A_{0} N$, then $x A_{0} \subset A_{0}$.

If $x A_{0} N \subset B_{0} M$, then $x A_{0} \subset B_{0} M$.

If $x B_{0} M \subset D$, then $x B_{0} \subset D$.

If $x B_{0} M \subset M$, then $x B_{0} \subset M$.

If $x B_{0} M \subset N$, then $x B_{0} \subset N$.

If $x B_{0} M \subset M N$, then $x B_{0} \subset M N$.

If $x B_{0} M \subset A_{0} N$, then $x B_{0} \subset A_{0} N$.

If $x B_{0} M \subset B_{0} M$, then $x B_{0} \subset B_{0}$. 
(4.5) Each $\star_{i}$ is a star operation on $D$. We have $\star_{1}=\mathrm{d}$ and $\star_{9}=\mathrm{v}$.

(4.6) $|\Sigma(D)|=9$.

(4.7) We have $\overline{\mathrm{F}}(D) \backslash \mathrm{F}(D)=\left\{x A, x B, x A N, x B M, x A B_{0}, x A_{0} B, K \mid x \in K \backslash\right.$ $\{0\}, A_{0}$ (resp. $\left.B_{0}\right)$ is a fractional ideal of $D$ with type $\alpha$ (resp. type $\beta$ ) $\}$.

(4.8) Let $\star$ be a semistar operation on $D$. Then we have that $A^{\star}$ is either $A$ or $K, B^{\star}$ is either $B$ or $K,(A N)^{\star}$ is either $A N$ or $A$ or $K$, and $(B M)^{\star}$ is either $B M$ or $B$ or $K$. If $A^{\star}=A$, then $\left(A B_{0}\right)^{\star}=A B_{0}$ for each fractional ideal $B_{0}$ of $D$ with type $\beta$, and if $B^{\star}=B$, then $\left(A_{0} B\right)^{\star}=A_{0} B$ for each fractional ideal $A_{0}$ of $D$ with type $\alpha$.

(4.9) Let $\star_{i}$ be a star operation on $D$.

(1) Set $I_{0}^{\star_{i}^{1}}=I_{0}^{\star_{i}}$ for each $I_{0} \in \mathrm{F}(D)$, and set $A^{\star_{i}^{1}}=A, B^{\star_{i}^{1}}=B,(A N)^{\star_{i}^{1}}=A N$ and $(B M)^{\star_{i}^{1}}=B M$. Then there is determined a unique mapping $\star_{i}^{1}$ from $\overline{\mathrm{F}}(D)$ to $\overline{\mathrm{F}}(D)$.

(2) Set $I_{0}^{\star_{i}^{2}}=I_{0}^{\star_{i}}$ for each $I_{0} \in \mathrm{F}(D)$, and set $A^{\star_{i}^{2}}=A, B^{\star_{i}^{2}}=B,(A N)^{\star_{i}^{2}}=A N$ and $(B M)^{\star_{i}^{2}}=B$. Then there is determined a unique mapping $\star_{i}^{2}$ from $\overline{\mathrm{F}}(D)$ to $\overline{\mathrm{F}}(D)$.

(3) Set $I_{0}^{\star_{i}^{3}}=I_{0}^{\star_{i}}$ for each $I_{0} \in \mathrm{F}(D)$, and set $A^{\star_{i}^{3}}=A, B^{\star_{i}^{3}}=B,(A N)^{\star_{i}^{3}}=A$ and $(B M)^{\star_{i}^{3}}=B M$. Then there is determined a unique mapping $\star_{i}^{3}$ from $\overline{\mathrm{F}}(D)$ to $\overline{\mathrm{F}}(D)$.

(4) Set $I_{0}^{\star_{i}^{4}}=I_{0}^{\star_{i}}$ for each $I_{0} \in \mathrm{F}(D)$, and set $A^{\star_{i}^{4}}=A, B^{\star_{i}^{4}}=B,(A N)^{\star_{i}^{4}}=A$ and $(B M)^{\star_{i}^{4}}=B$. Then there is determined a unique mappping $\star_{i}^{4}$ from $\overline{\mathrm{F}}(D)$ to $\overline{\mathrm{F}}(D)$.

(5) Set $I_{0}^{\star_{i}^{5}}=I_{0}^{\star_{i}}$ for each $I_{0} \in \mathrm{F}(D)$, and set $A^{\star_{i}^{5}}=A, B^{\star_{i}^{5}}=K$ and $(A N)^{\star_{i}^{5}}=$ $A N$. Then there is determined a unique mapping $\star_{i}^{5}$ from $\overline{\mathrm{F}}(D)$ to $\overline{\mathrm{F}}(D)$.

(6) Set $I_{0}^{\star_{i}^{6}}=I_{0}^{\star_{i}}$ for each $I_{0} \in \mathrm{F}(D)$, and set $A^{\star_{i}^{6}}=A, B^{\star_{i}^{6}}=K$ and $(A N)^{\star_{i}^{6}}=A$. Then there is determined a unique mapping $\star_{i}^{6}$ from $\overline{\mathrm{F}}(D)$ to $\overline{\mathrm{F}}(D)$.

(7) Set $I_{0}^{\star_{i}^{7}}=I_{0}^{\star_{i}}$ for each $I_{0} \in \mathrm{F}(D)$, and set $A^{\star_{i}^{7}}=K, B^{\star_{i}^{7}}=B$ and $(B M)^{\star_{i}^{7}}=$ $B M$. Then there is determined a unique mapping $\star_{i}^{7}$ from $\overline{\mathrm{F}}(D)$ to $\overline{\mathrm{F}}(D)$.

(8) Set $I_{0}^{\star_{i}^{8}}=I_{0}^{\star_{i}}$ for each $I_{0} \in \mathrm{F}(D)$, and set $A^{\star_{i}^{8}}=K, B^{\star_{i}^{8}}=B$ and $(B M)^{\star_{i}^{8}}=B$. Then there is determined a unique mapping $\star_{i}^{8}$ from $\overline{\mathrm{F}}(D)$ to $\overline{\mathrm{F}}(D)$.

(9) Set $I_{0}^{\star_{i}^{9}}=I_{0}^{\star_{i}}$ for each $I_{0} \in \mathrm{F}(D)$, and set $A^{\star_{i}^{9}}=K$ and $B^{\star_{i}^{9}}=K$. Then there is determined a unique mapping $\star_{i}^{9}$ from $\overline{\mathrm{F}}(D)$ to $\overline{\mathrm{F}}(D)$.

(4.10) Each $\star_{i}^{j}$ satisfies the following conditions: $(x I)^{\star}=x I^{\star}$ for each $x \in K \backslash\{0\}$ and $I \in \overline{\mathrm{F}}(D) ; I \subset I^{\star}$ for each $I \in \overline{\mathrm{F}}(D)$; and $\left(I^{\star}\right)^{\star}=I^{\star}$ for each $I \in \overline{\mathrm{F}}(D)$.

(4.11) Let $x \in K \backslash\{0\}$, and let $A_{0}$ (resp. $B_{0}$ ) be a fractional ideal of $D$ with type $\alpha$ (resp. type $\beta$ ).

If $x M \subset A N$, then $(x) \subset A N$.

If $x M \subset B M$, then $(x) \subset B$.

If $x M \subset A B_{0}$, then $(x) \subset A B_{0}$.

If $x M \subset A_{0} B$, then $(x) \subset A_{0} B$. 


$$
\begin{aligned}
& \text { If } x N \subset A N \text {, then }(x) \subset A \text {. } \\
& \text { If } x N \subset B M \text {, then }(x) \subset B M \text {. } \\
& \text { If } x N \subset A B_{0} \text {, then }(x) \subset A B_{0} \text {. } \\
& \text { If } x N \subset A_{0} B \text {, then }(x) \subset A_{0} B \text {. } \\
& \text { If } x M N \subset A N \text {, then }(x) \subset A \text {. } \\
& \text { If } x M N \subset B M \text {, then }(x) \subset B \text {. } \\
& \text { If } x M N \subset A B_{0} \text {, then }(x) \subset A B_{0} \text {. } \\
& \text { If } x M N \subset A_{0} B \text {, then }(x) \subset A_{0} B \text {. } \\
& \text { If } x A_{0} N \subset A N \text {, then } x A_{0} \subset A \text {. } \\
& \text { If } x A_{0} N \subset B M \text {, then } x A_{0} \subset B M \text {. } \\
& \text { If } x A_{0} N \subset A B_{0} \text {, then } x A_{0} \subset A B_{0} \text {. } \\
& \text { If } x A_{0} N \subset A_{0} B \text {, then } x A_{0} \subset A_{0} B \text {. } \\
& \text { If } x B_{0} M \subset A N \text {, then } x B_{0} \subset A N \text {. } \\
& \text { If } x B_{0} M \subset B M \text {, then } x B_{0} \subset B \text {. } \\
& \text { If } x B_{0} M \subset A B_{0} \text {, then } x B_{0} \subset A B_{0} \text {. } \\
& \text { If } x B_{0} M \subset A_{0} B \text {, then } x B_{0} \subset A_{0} B \text {. }
\end{aligned}
$$

(4.12) Let $I \in \mathrm{F}(D)$ and $J \in \overline{\mathrm{F}}(D)$ such that $I \subset J$. Then each member in $\left\{\star_{1}^{1}, \star_{1}^{2}, \star_{2}^{2}, \star_{7}^{2}, \star_{1}^{3}, \star_{3}^{3}, \star_{5}^{3}, \star_{1}^{4}, \star_{2}^{4}, \star_{3}^{4}, \star_{4}^{4}, \star_{5}^{4}, \star_{6}^{4}, \star_{7}^{4}, \star_{8}^{4}, \star_{9}^{4}, \star_{1}^{5}, \star_{2}^{5}, \star_{7}^{5}, \star_{1}^{6}, \star_{2}^{6}, \star_{3}^{6}, \star_{4}^{6}, \star_{5}^{6}, \star_{6}^{6}, \star_{7}^{6}\right.$, $\left.\star_{8}^{6}, \star_{9}^{6}, \star_{1}^{7}, \star_{3}^{7}, \star_{5}^{7}, \star_{1}^{8}, \star_{2}^{8}, \star_{3}^{8}, \star_{4}^{8}, \star_{5}^{8}, \star_{6}^{8}, \star_{7}^{8}, \star_{8}^{8}, \star_{9}^{8}, \star_{1}^{9}, \star_{2}^{9}, \star_{3}^{9}, \star_{4}^{9}, \star_{5}^{9}, \star_{6}^{9}, \star_{7}^{9}, \star_{8}^{9}, \star_{9}^{9}\right\}$ is monotone for $I \subset J$.

(4.13) (1) Each of $\star_{2}^{1}, \star_{7}^{1}, \star_{2}^{3}, \star_{4}^{3}, \star_{6}^{3}, \star_{7}^{3}, \star_{8}^{3}, \star_{9}^{3}, \star_{2}^{7}, \star_{4}^{7}, \star_{6}^{7}, \star_{7}^{7}, \star_{8}^{7}, \star_{9}^{7}$ is not monotone for $B_{0} M \subset B M$.

(2) Each of $\star_{3}^{1}, \star_{4}^{1}, \star_{5}^{1}, \star_{6}^{1}, \star_{8}^{1}, \star_{9}^{1}, \star_{3}^{2}, \star_{4}^{2}, \star_{5}^{2}, \star_{6}^{2}, \star_{8}^{2}, \star_{9}^{2}, \star_{3}^{5}, \star_{4}^{5}, \star_{5}^{5}, \star_{6}^{5}, \star_{8}^{5}, \star_{9}^{5}$ is not monotone for $A_{0} N \subset A N$.

(4.14) Let $A_{0}$ (resp. $\left.B_{0}\right)$ be a fractional ideal of $D$ with type $\alpha$ (resp. type $\beta$ ), and let $x \in K \backslash\{0\}$.

If $x A N \subset A$, then $x A \subset A$.

If $x A N \subset A B_{0}$, then $x A \subset A B_{0}$.

If $x B M \subset B$, then $x B \subset B$.

If $x B M \subset A_{0} B$, then $x B \subset A_{0} B$.

(4.15) Each member in $\left\{\star_{1}^{1}, \star_{1}^{2}, \star_{2}^{2}, \star_{7}^{2}, \star_{1}^{3}, \star_{3}^{3}, \star_{5}^{3}, \star_{1}^{4}, \star_{2}^{4}, \star_{3}^{4}, \star_{4}^{4}, \star_{5}^{4}, \star_{6}^{4}, \star_{7}^{4}, \star_{8}^{4}, \star_{9}^{4}\right.$, $\star_{1}^{5}, \star_{2}^{5}, \star_{7}^{5}, \star_{1}^{6}, \star_{2}^{6}, \star_{3}^{6}, \star_{4}^{6}, \star_{5}^{6}, \star_{6}^{6}, \star_{7}^{6}, \star_{8}^{6}, \star_{9}^{6}, \star_{1}^{7}, \star_{3}^{7}, \star_{5}^{7}, \star_{1}^{8}, \star_{2}^{8}, \star_{3}^{8}, \star_{4}^{8}, \star_{5}^{8}, \star_{6}^{8}, \star_{7}^{8}, \star_{8}^{8}, \star_{9}^{8}, \star_{1}^{9}, \star_{2}^{9}$, $\left.\star_{3}^{9}, \star_{4}^{9}, \star_{5}^{9}, \star_{6}^{9}, \star_{7}^{9}, \star_{8}^{9}, \star_{9}^{9}\right\}$ is a semistar operation on $D$. We have $\star_{1}^{1}=\mathrm{d}^{\prime}$ and $\star_{9}^{9}=\mathrm{v}^{\prime}$.

(4.16) We have $\left|\Sigma^{\prime}(D)\right|=54$.

\section{REFERENCES}

$[\mathrm{H}] \mathrm{W}$. Heinzer, Integral domains in which each non-zero ideal is divisorial, Math. 15 (1968), 164-170.

[M1] R. Matsuda, Note on the number of semistar-operations, Math. J. Ibaraki Univ. 31 (1999), 47-53. 
[M2] R. Matsuda, Note on the number of semistar operations, VIII, Math. J. Ibaraki Univ. 37 (2005), 53-79. 